\title{
Turbulence in an autooscillating complex plasma
}

\author{
Mierk Schwabe, Sergey Zhdanov, and Christoph Räth
}

\begin{abstract}
We present an experiment on the development of turbulence in a microparticle cloud in a low temperature plasma laboratory on board the International Space Station. First, the complex plasma cloud was stabilized with an alternating electric field. Once the stabilization was switched off, a so-called heartbeat instability developed in which the microparticles are oscillating radially. We show that the kinetic energy spectra develop a double cascade once the cloud becomes unstable. A double cascade has been predicted by Kraichnan and Leith for forced twodimensional turbulence, but has only seldomly been observed in experiments. We also show that the reduced rates of energy and enstrophy transfer can be used to infer the excitation wave number $k_{\text {exc }}$, in good agreement with $k_{\text {exc }}$ obtained from the energy spectrum.
\end{abstract}

Index Terms-Dusty plasma, complex plasma, turbulence, instability, double cascade

\section{INTRODUCTION}

$\mathbf{T}$ URBULENCE is one of the well-known outstanding issues of modern theoretical physics, despite being a common and in many cases key phenomenon. Although turbulence is a well-studied subject, even the definition of what constitutes a turbulent flow is not commonly agreed upon [1]. Turbulence is often defined by its main features, for instance, fluid motion on many scales, and a large increase in flow resistance compared to an imaginary laminar flow under the same conditions [2]. Turbulence also occurs in systems not typically expected to be turbulent, for instance viscoelastic fluid flows with low Reynolds numbers [2]-[6].

In experiments on turbulence in conventional liquids, the carriers of the interaction are not directly observable. Instead, typically tracer particles are used - a method that might not produce reliable results [7]. Commonly, energy spectra are used to study turbulence. They normally follow a powerlaw dependence $E \propto k^{-n}$, where $n$ is the spectral index and $k$ the wave number. In three dimensions, the energy spectrum typically shows a $E \propto k^{-5 / 3}$ dependence [8]. In two dimensions, a double cascade can develop when the turbulence is forced [9], [10], which means that the spectrum splits into two regimes: At wave numbers that are smaller than the excitation wavenumer $k<k_{\text {exc }}$ (larger spatial scales), the "inverse cascade" develops with a spectral index of $n=5 / 3$, and at larger wave numbers $k>k_{\text {exc }}$ (smaller spatial scales), the "direct cascade" with $n=3$ appears. Fig. 1 schematically depicts this double cascade. The energy flux is mainly directed towards lower wave numbers (larger spatial scale) in the inverse cascade range, and the vorticity mainly flows to larger wave numbers (smaller spatial scale) in the direct cascade.

The authors are with the Institut für Materialphysik im Weltraum, Deutsches Zentrum für Luft- und Raumfahrt (DLR), Münchener Str. 22, 82234 Weßling, Germany.

Manuscript received Jun 22, 2017; revised Aug 22, 2017

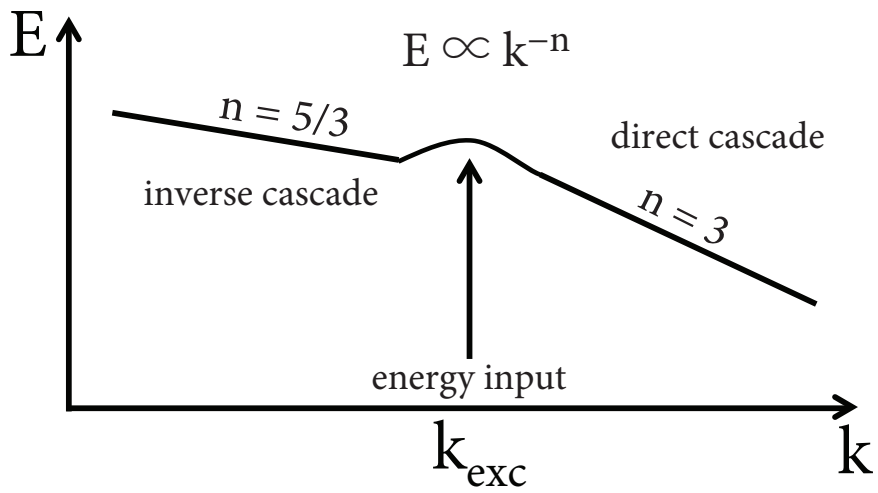

Fig. 1. The double cascade in forced two-dimensional turbulence.

The proportionality factors $C_{\mathrm{i}}$ and $C_{\mathrm{d}}$ connect the energy to the wave number

$$
\begin{aligned}
& E=C_{\mathrm{i}}^{2 / 3} k^{-5 / 3} \\
& E=C_{\mathrm{d}}^{2 / 3} k^{-3} .
\end{aligned}
$$

They are proportional to the rates of transfer of kinetic energy resp. mean-square vorticity (enstrophy) in the inverse/direct cascade ranges. We will refer to $C_{\mathrm{i} / \mathrm{d}}$ as reduced rates of transfer. The wave number where the inverse and the direct range merge is exactly the wave number corresponding to the scale of energy input into the system, $k_{\text {exc }}$, see Fig. 1.

It is difficult to observe both ranges of the double cascade simultaneously due to the large range of scales that needs to be covered, and this has only seldomly been achieved in experiments or simulations [11].

Here, we present an experiment performed in the PK3 Plus Laboratory on board the International Space Station (ISS) [12]-[14] which allows directly investigating the role of the fundamental particles as the carriers of the interaction in turbulence [15]-[22]. We study microparticles immersed in a low temperature plasma, a so-called complex plasma. The microparticles acquire high negative charges and interact with each other. Due to their relatively large size and slow velocities, their dynamics can be observed in real time. This makes possible an investigation on the most basic level - that of the individual particles.

\section{EXPERIMENTAL SETUP}

The PK-3 Plus Laboratory was hosted on board the ISS from $2005-2013$ and operated for 21 missions with three or four experimental runs of approximately 90 minutes in each mission. Performing experiments under microgravity conditions is essential for studies of extended three-dimensional microparticle clouds immersed in a low-temperature plasma. 


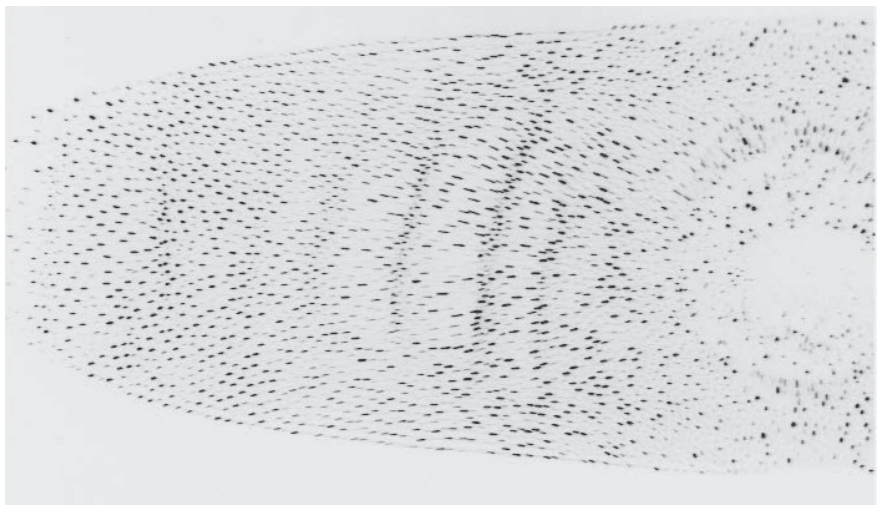

Fig. 2. Inverted experimental image with field of view $35 \times 18 \mathrm{~mm}^{2}$. The black dots are images of microparticles. The central void is visible as particlefree region in the right of the image. Wave ridges (leftwards propagating) called "oscillons" [23] can be seen in the middle part of the microparticle cloud to the left of the void.

In the experiment presented here [18], [22], [23], we analyzed data obtained with the quadrant view camera at a resolution of approximately $50 \mu \mathrm{m} / \mathrm{pixel}$ and a frame rate of 50 frames per second (fps). This camera observes the left part of the microparticle cloud up to the central region where a microparticle-free void typically forms. The void formation is caused by the interplay of the ion drag force pushing the microparticles from the chamber center towards the chamber edges, as well as the interparticle repulsion and the plasma electric force acting in the direction opposite to that of the ion drag force [24].

The experiment was performed in an argon plasma at a pressure of $9 \mathrm{~Pa}$. Melamine-formaldehyde (MF) microparticles of $9.2 \mu \mathrm{m}$ diameter and a mass density of $1510 \mathrm{~kg} \mathrm{~m}^{-3}$ were inserted into the plasma. Figure 2 shows the inverse of a still image obtained with the quadrant view camera. Every dot represents a microparticle. Note the void in the right of the image.

At the beginning of the experiment, the plasma was stabilized by applying a relatively slowly alternating electric field in addition to the radio-frequency field sustaining the plasma. The electric field alternated at frequencies between 42 and $51 \mathrm{~Hz}$, which is too fast for the microparticles to follow, but slow enough for plasma ions and electrons. The combined effect of the electric field and that of the streaming plasma ions was to close the central microparticle-free void, and to prevent the onset of an instability. Once the stabilization was turned off, the so-called heartbeat-instability developed - a regular, heartbeat like expansion and contraction of the void. The frequency of this pulsation in the experiment presented here was $f_{\mathrm{HB}}=2.8 \mathrm{~Hz}$. The heartbeat instability is thought to be caused by the periodic formation of a sheath at the void boundary [25].

To study turbulence, we use the heartbeat to "stir" the microparticles and to inject energy. The movement of the void boundary leads to regular movement of the whole microparticle cloud, and to the formation of slowly drifting wave ridges which we term "oscillons". The formation of the oscillons is the result of a modulational instability [23]. The

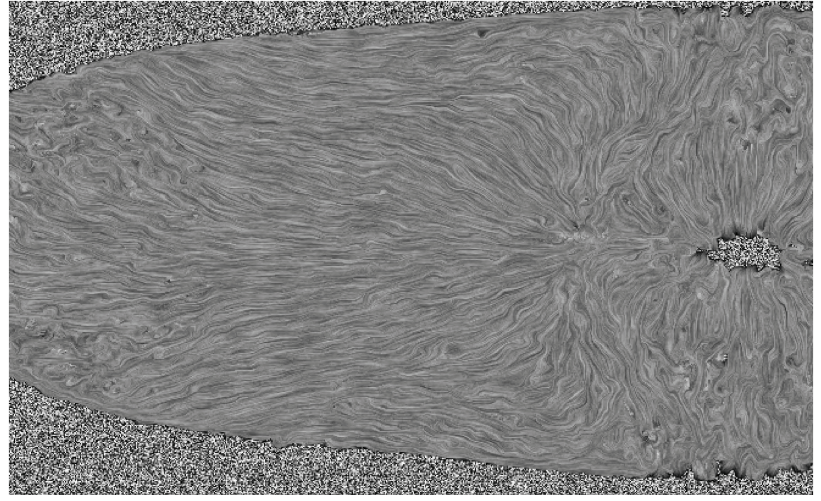

Fig. 3. Flow field of the microparticles produced with line integral convolution [27], calculated by averaging over a time period of $5 \mathrm{~s}$. Field of view: $33 \times 18 \mathrm{~mm}^{2}$. Note the vortices with various sizes, see [22].

oscillons are well visible as vertically extended denser regions in Figure 2. On average, the microparticles move outwards in the central part of the cloud, and inwards along the edges, reminding of dipole-type large vortices that are often present in weightless complex plasma clouds [26]. We believe that the heartbeat channels the microparticle movement mainly in two dimensions, i.e. radially and axially, but we cannot exclude a flow in the third (azimuthal) direction, as we are unable to trace particles moving that way.

Figure 3 shows the average flowfield. This image was produced by calculating velocity maps, i.e. the average spatial distributions of the horizontal $\left(v_{\mathrm{x}}\right)$ and vertical $\left(v_{\mathrm{y}}\right)$ velocities. Then we used the method of line integral convolution [27]: A random background pattern was distorted along the vectors defined by the velocity maps. Apart from the general radial movement, vortices on several scales are visible, as is expected for turbulence.

\section{ANALYSIS}

We are interested in studying the evolution of the energy spectra before, during and after the onset of the instability. In order to obtain the energy spectra, we first measure particle velocities. Next, we calculate velocity maps $v_{\mathrm{x}}(x, y)$ and $v_{\mathrm{y}}(x, y)$ for every recorded frame by averaging velocities in a sliding window encompassing a time period of $5 \mathrm{~s}$ and a radius of 5 pixel. The size of the sliding window was determined as optimal to still include dynamical effects while minimizing the influence of missing data in the velocity maps [22], [28]. Next, we Fourier-transform the velocity maps:

$$
\begin{aligned}
& \widetilde{v_{\mathrm{x}}}\left(k_{\mathrm{x}}, k_{\mathrm{y}}\right)=\operatorname{FFT}\left(v_{\mathrm{x}}(x, y)\right) \\
& \widetilde{v}_{\mathrm{y}}\left(k_{\mathrm{x}}, k_{\mathrm{y}}\right)=\operatorname{FFT}\left(v_{\mathrm{y}}(x, y)\right) .
\end{aligned}
$$

The map of the kinetic energy in k-space is then given by

$$
E\left(k_{\mathrm{x}}, k_{\mathrm{y}}\right)={\widetilde{v_{\mathrm{x}}}}^{2}\left(k_{\mathrm{x}}, k_{\mathrm{y}}\right)+{\widetilde{v_{\mathrm{y}}}}^{2}\left(k_{\mathrm{x}}, k_{\mathrm{y}}\right) .
$$

In order to obtain the energy for a specific wave vector modulus range $|k| \ldots|k|+|d k|$, we calculate the average of all energies $\mathrm{E}\left(k_{\mathrm{x}}, k_{\mathrm{y}}\right)$ with $\left(k_{\mathrm{x}}, k_{\mathrm{y}}\right)$ values matching the given $|k|$-range. 


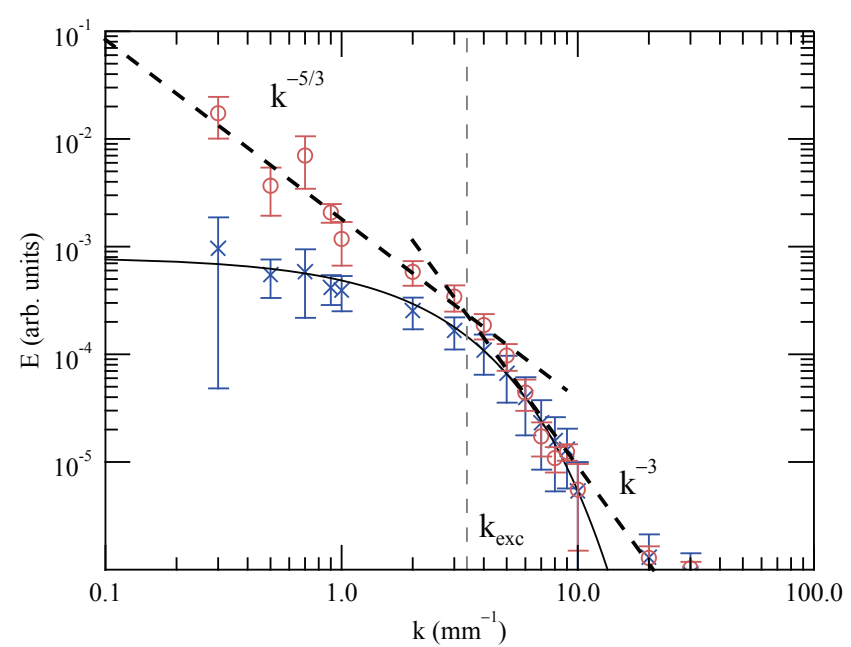

Fig. 4. Comparison of spectra before (crosses) and after (circles) the onset of the instability. The thick dashed lines show dependencies with spectral indices $n=3$ and $n=5 / 3$ as guides to the eye. The vertical thin dashed line indicates the excitation wave number $k_{\text {exc }}$. Following [22].

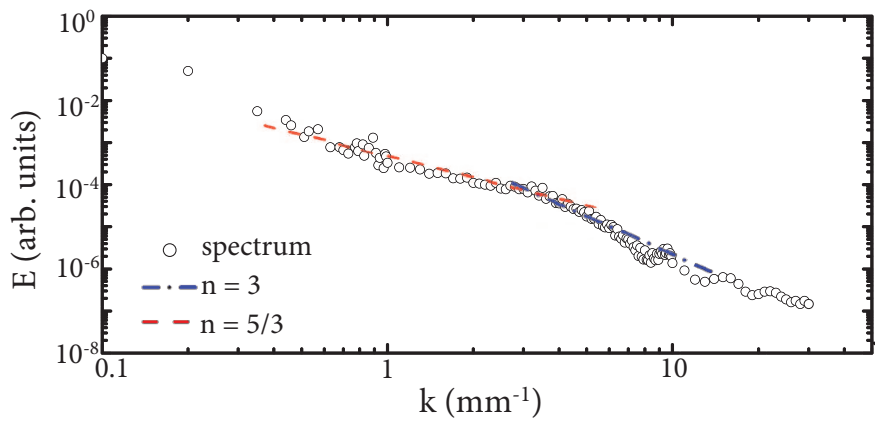

Fig. 5. Kinetic energy spectrum for fully developed turbulence. Ranges with spectral indices $n=3$ and $n=5 / 3$ are marked as guides to the eye. Following [18].

\section{ENERGY SPECTRA}

Figures 4 and 5 show energy spectra obtained at three points in time: 1) Before the onset of the instability (Fig. 4, crosses), 2) within the first $50 \mathrm{~s}$ after the onset of the instability (Fig. 4, circles), and 3) for fully developed turbulence (Fig. 5). Before the onset of the instability, the energy spectrum is best fitted with an exponential dependence. Exponential energy spectra can occur in laminar flows and in flows with strong dissipation [29], [30]. After the onset, the spectrum changes dramatically at small wave numbers: The energy rises, as is expected due to the energy input from the heartbeat. But, interestingly, its characteristic changes to a power-law close to $E \propto k^{-5 / 3}$, as predicted for the inverse cascade range in forced two-dimensional turbulence. At larger wave numbers, when a power law is fitted, the dependence is close to the $E \propto k^{-3}$ predicted for the direct cascade range. Note that there is no significant difference between the spectra before and after the instability onset for large wave numbers. Also in the case of fully developed turbulence, the two ranges of the spectrum are well visible, see Fig. 5, and [18] for details.

Fitting this double spectrum separately for the ranges $0.3 \leq$ $k \leq 4 \mathrm{~mm}^{-1}$ and $4 \leq k \leq 15 \mathrm{~mm}^{-1}$ in Fig. 4 results in $n=$ $1.5 \pm 0.5$ and $n=3.1 \pm 0.3$ for the two ranges, respectively. These values fit the predicted $n=5 / 3$ and $n=3.0$ within the uncertainties.

\section{DETERMINING THE EXCITATION WAVE NUMBER}

There are several methods to determine the excitation wave number $k_{\text {exc }}$. First of all, considering the fact that the energy input stems from the heartbeat instability, we can estimate the expected excitation wave number as

$$
k_{\mathrm{exc}}=\frac{2 \pi f_{\mathrm{HB}}}{C_{\mathrm{DAW}}},
$$

where $f_{\mathrm{HB}}=2.8 \mathrm{~Hz}$ denotes the heartbeat frequency and $C_{\text {DAW }} \approx 6.5 \mathrm{~mm} / \mathrm{s}$ is the microparticle speed of sound [23]. This results in an expected value of the excitation wave number $k_{\mathrm{exc}} \approx 2.7 \mathrm{~mm}^{-1}$.

Assuming that the observed double cascade is actually that predicted by Kraichnan's and Leith's theory, we use the fact that the excitation wave number is just that wave number where the inverse and direct ranges of the spectrum meet. Looking at Fig. 4 and 5, we determine $k_{\mathrm{exc}} \approx 3.3 \mathrm{~mm}^{-1}$.

Under the same assumptions, we can alternatively employ the fact that the energy values determined using equations (1) and (2) are equal at $k=k_{\text {exc }}$. Thus, we can obtain the excitation wave number by measuring the reduced rates of transfer in the two ranges, $C_{\mathrm{i}}$ and $C_{\mathrm{d}}$. We select wave numbers $k_{\mathrm{i}}=2 \mathrm{~mm}^{-1}$ and $k_{\mathrm{d}}=6 \mathrm{~mm}^{-1}$ as representative wave numbers in the inverse respectively direct range, and determine the reduced rates of transfer at these wave numbers by using equations (1) and (2). The excitation wave number is then obtained by equating (1) and (2)

$$
C_{\mathrm{i}}^{2 / 3} k_{\mathrm{exc}}^{-5 / 3}=C_{\mathrm{d}}^{2 / 3} k_{\mathrm{exc}}^{-3} .
$$

This results in

$$
k_{\mathrm{exc}}=\sqrt{C_{\mathrm{d}} / C_{\mathrm{i}}}=3.9 \pm 0.5 \mathrm{~mm}^{-1}
$$

which agrees well with that determined from the point where the two ranges meet, and is just slightly larger than that calculated from the heartbeat frequency and microparticle speed of sound.

\section{Discussion AND CONCLUSION}

It is curious that we observe a double cascade in the kinetic energy spectrum that seemingly fits that predicted for forced two-dimensional turbulence. Recall that the original work by Kraichnan assumed an inviscid two-dimensional flow. In our experiment, the microparticles experience friction from the background gas and viscosity from the interaction with neighboring microparticles. Also, we cannot exclude a particle flow in the third dimension, especially at short scales, even though it seems that the heartbeat instability channels the flow radially. Nevertheless, the spectral indices that we obtain from fitting the spectra agree well with those predicted by Kraichnan and Leith, and the excitation wave number obtained under the assumption that their theory is applicable is very close to that obtained from the heartbeat frequency and microparticle 
velocity of sound. Clearly, further work to investigate this coincidence is warranted. Thus, complex plasmas have the potential to serve as an exciting new tool to study turbulence with the unique capability to directly follow the motion of the carriers of the interaction.

\section{ACKNOWLEDGMENT}

We would like to thank Dr. Hubertus Thomas for helpful discussion, and the PK-3 Plus team at the Joint Institute of High Temperatures, Russian Academy of Sciences, Moscow, and at the Institute of Materials Physics in Space, DLR Oberpfaffenhofen, Germany, for providing us with the data. PK-3 Plus was funded by DLR/BMWi under contract No. 50WM1203.

\section{REFERENCES}

[1] D. J. Tritton, Physical Fluid Dynamics. Clarendon, 1988.

[2] A. Groisman and V. Steinberg, "Elastic turbulence in a polymer solution flow," Nature, vol. 405, pp. 53-55, 2000

[3] — "Elastic turbulence in curvilinear flows of polymer solutions," New J. Phys., vol. 6, p. 29, 2004.

[4] J. Mitchell, K. Lyons, A. M. Howe, and A. Clarke, "Viscoelastic polymer flows and elastic turbulence in three-dimensional porous structures," Soft Matter, vol. 12, 2016.

[5] P. Ballesta and M. A. Alves, "Purely elastic instabilities in a microfluidic flow focusing device," Phys. Rev. Fluids, vol. 2, p. 053301, 2017.

[6] A. V. Malm and T. A. Waigh, "Elastic turbulence in entangled semidilute dna solutions measured with optical coherence tomography velocimetry," Sci. Rep., vol. 7, 2017.

[7] V. Mathai, E. Calzavarini, J. Brons, C. Sun, and D. Lohse, "Microbubbles and microparticles are not faithful tracers of turbulent acceleration," Phys. Rev. Lett., vol. 117, p. 024501, 2016.

[8] U. Frisch, Turbulence : the legacy of A.N. Kolmogorov. Cambridge University Press, 1995.

[9] R. H. Kraichnan, "Inertial range in two dimensional turbulence," Phys. Fluids, vol. 10, p. 1417, 1967.

[10] C. E. Leith, "Diffusion approximation for two-dimensional turbulence," Phys. Fluids, vol. 11, p. 671, 1968

[11] G. Boffetta and R. E. Ecke, "Two-dimensional turbulence," Ann. Rev. Fluid Mech., vol. 44, pp. 427-451, 2012.

[12] H. M. Thomas, G. E. Morfill, V. E. Fortov, et al., "Complex plasma laboratory pk-3 plus on the international space station," New J. Phys., vol. 10, p. 033036, 2008.

[13] V. I. Molotkov, H. M. Thomas, A. M. Lipaev, V. N. Naumkin, A. V. Ivlev, and S. A. Khrapak, "Complex (dusty) plasma research under microgravity conditions: Pk-3 plus laboratory on the international space station," Int. J. Microgravity Sci. Appl., vol. 3, no. 35, p. 320302, 2015.

[14] A. G. Khrapak, V. I. Molotkov, A. M. Lipaev, et al., "Complex plasma research under microgravity conditions: Pk-3 plus laboratory on the international space station," Contrib. Plasma Phys., vol. 56, pp. $253-$ 262, 2016.

[15] Y.-Y. Tsai, M.-C. Chang, and L. I, "Observation of multifractal intermittent dust-acoustic-wave turbulence," Phys. Rev. E, vol. 86, p. 045402, 2012.

[16] M. Schwabe, S. Zhdanov, C. Räth, D. B. Graves, H. M. Thomas, and G. E. Morfill, "Collective effects in vortex movement in complex plasmas," Phys. Rev. Lett., vol. 112, p. 115002, 2014.

[17] A. Gupta, R. Ganesh, and A. Joy, "Kolmogorov flow in two dimensional strongly coupled dusty plasma," Phys. Plasmas, vol. 21, p. 073707, 2014.

[18] S. Zhdanov, M. Schwabe, C. Räth, H. M. Thomas, and G. E. Morfill, "Wave turbulence observed in an auto-oscillating complex (dusty) plasma," EPL, vol. 110, p. 35001, 2015.

[19] R. A. Galvão and L. F. Ziebell, "Weak turbulence in dusty plasmas with collisional dust charging: Quasilinear wave-particle interaction," Phys. Rev. E, 2015.

[20] S. K. Tiwari, V. S. Dharodi, A. Das, B. G. Patel, and P. Kaw, "Turbulence in strongly coupled dusty plasmas using generalized hydrodynamic description," Phys. Plasmas, 2015.

[21] S. Zhdanov, C.-R. Du, M. Schwabe, V. Nosenko, H. M. Thomas, and G. E. Morfill, "Wake turbulence observed behind an upstream "extra" particle in a complex (dusty) plasma," EPL, vol. 114, p. 55002, 2016.
[22] M. Schwabe, S. Zhdanov, and C. Räth, "Instability onset and scaling laws of an autooscillating turbulent flow in a complex (dusty) plasma," Phys. Rev. E, vol. 95, p. 041201(R), 2017.

[23] S. K. Zhdanov, M. Schwabe, R. Heidemann, R. Sütterlin, H. M. Thomas, M. Rubin-Zuzic, H. Rothermel, T. Hagl, A. V. Ivlev, G. E. Morfill, V. I. Molotkov, A. M. Lipaev, O. F. Petrov, V. E. Fortov, and T. Reiter, "Autooscillations in complex plasmas," New J. Phys., vol. 12, p. 043006, 2010.

[24] J. Goree, G. E. Morfill, V. N. Tsytovich, and S. V. Vladimirov, "Theory of dust voids in plasmas," Phys. Rev. E, vol. 59, no. 6, pp. 7055-7067, 1999.

[25] M. Y. Pustylnik, A. V. Ivlev, N. Sadeghi, R. Heidemann, S. Mitic, H. M. Thomas, and G. E. Morfill, "On the heterogeneous character of the heartbeat instability in complex (dusty) plasmas," Phys. Plasmas, vol. 19, p. 103701, 2012.

[26] M. Schwabe and D. B. Graves, "Simulating the dynamics of complex plasmas," Phys. Rev. E, vol. 88, p. 023101, 2013.

[27] B. Cabral and L. C. Leedom, "Imaging vector fields using line integral convolution," in Proceedings of the 20th annual conference on Computer graphics and interactive techniques, 1993.

[28] P. Arévalo, E. Churazov, I. Zhuravleva, C. Hernández-Monteagudo, and M. Revnivtsev, "A mexican hat with holes: calculating low-resolution power spectra from data with gaps," Mon. Not. R. Astron. Soc., vol. 426, pp. 1793-1807, 2012.

[29] M. K. Verma, "Variable enstrophy flux and energy spectrum in twodimensional turbulence with Ekman friction", EPL 98, 14003, 2012.

[30] K. S. Reddy, and M. K. Verma, "Strong anisotropy in quasi-steady magnetohydrodynamic turbulence for high interaction parameters," Phys. Fluids, vol. 26, p. 025109, 2014.

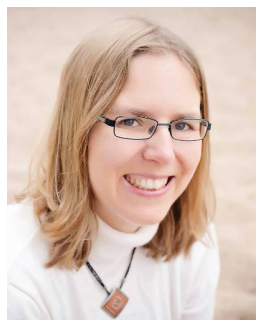

Mierk Schwabe received the diploma in physics from the Technical University, Munich in 2006 and the Ph.D. degree in physics from the LudwigMaximilians-Universität, Munich in 2009. Previously, she was with the Max-Planck-Institute for Extraterrestrial Physics in Garching, Germany, and the University of California at Berkeley, CA She is currently with the Institute of Materials Physics in Space, German Aerospace Center, Oberpfaffenhofen. Her research interests include experiments and simulations of waves and instabilities, turbulence, and the emergence of collaborative effects in complex plasmas.

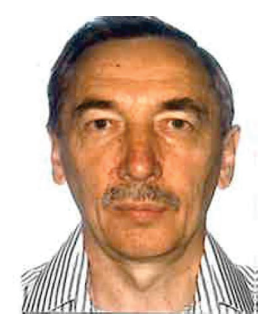

Sergey Zhdanov is currently a Researcher at the Institute of Materials Physics in Space, German Aerospace Center, Oberpfaffenhofen (DLR). His main research interests include self-organization, transport and delicate dynamical phenomena in complex plasmas.

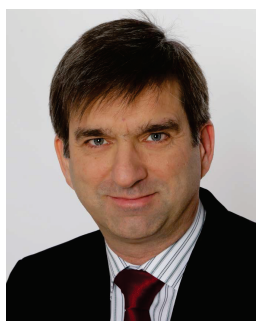

Christoph Räth received both the diploma and the $\mathrm{PhD}$ in physics from the Ludwig-MaximiliansUniversität, Munich, Germany, in 1994 and 1998 respectively. He was with the Max-Planck-Institute for Extraterrestrial Physics (MPE), Garching, Germany from 1998 until 2013. Since 2014 he works at the German Aerospace Center (DLR) and is currently with the Institute of Materials Physics in Space in Oberpfaffenhofen. His main research interests are complex systems and their analyses with (higher order) statistical measures. He is particularly interested in phenomena of self-organization and phase transition. His research activities also encompass astrophysics and medical physics. 